Methods A retrospective notes review was undertaken for referrals received in the first 4 weeks of the pandemic after 'lockdown' in UK. Numbers were compared to 2019. Information was sought on reason for referral and resulting actions.

Results 92 referrals were received, an increase of $26 \%$ from 2019. 11 patients died before first assessment, 2 declined a visit because of self isolation and 1 , no longer needed CPCT input. $40 \%$ referrals were from primary care, 33\% from specialty nurses, $13 \%$ from palliative care hospital teams, $7 \%$ from oncology and 5 others from secondary care. $78 \%$ had cancer, $14 \%$ - frailty, 5\% - dementia and the remainder end stage liver and heart disease. Referral information for the 79 patients reviewed cited pain in 24\%, 'support' (not specified) $-18 \%$, advance care planning (ACP) mentioned specifically in 14\%. Covid 19 affecting usual treatment or follow up plans leading to referral was mentioned in $27 \%$. On first review, $65 \%$ needed some aspect of ACP, $43 \%$ had pain, $82 \%$ had other symptom needs, $15 \%$-ADL needs, $20 \%$ - mood problems, $18 \%$ care or financial needs, $11 \%$ were in the last days of life.

Conclusions Although alternative health care delivery was imperative at the time to ensure hospital capacity, the impact on care delivered by CPCT cannot be underestimated. Referrals increased, $27 \%$ appearing to be as a direct result of altered services elsewhere. There is a need to work collaboratively across all settings as future surges of the pandemic progress.

\section{THE DEVELOPMENT AND EVALUATION OF A VIRTUAL VISITING PROGRAMME FACILITATING REGULAR FRIENDS AND FAMILY CONTACT FOR HOSPITAL INPATIENTS DURING THE COVID-19 PANDEMIC}

George Thomas, Rachel Rajadurai, Charlotte Browne, Natasha Wiggins, Tania Elias. Great Western Hospitals NHS Foundation Trust

\subsection{6/spcare-2021-PCC.32}

Background The ongoing COVID-19 pandemic has necessitated significant restrictions on physical visitors to hospitals to reduce infection risks. Consequently, patients are separated from loved ones at times of extreme stress and ill health. To reduce this burden, we have developed a structured service of staff-facilitated 'virtual visiting', where patients can see and communicate with loved ones using secure video calling applications on Trust electronic devices.

Methods Trust devices (Ipads and Iphones) are utilised by ward staff to facilitate virtual visits. Timing of such visits are usually pre-arranged with family members and patients after appropriate consent is gained. An instruction sheet was available to assist facilitators.

Quantitative and qualitative feedback was obtained focusing on the experiences of staff members who facilitated the visits, as well as some of the people 'visiting' during these calls.

Results 46 responses were received from staff facilitators, and from 11 virtual visitors. Unanimously $(n=46)$, staff indicated that virtual visiting helped them deliver good patient care and they would facilitate again. There was agreement that it added value for patients and staff $(n=46)$. Feedback from relatives reflected they found it very positive and it allowed them to know much better what was going on with their loved ones $(n=11)$.

The most common challenges included poor internet connections (40\%), lack of training resources (35\%), and lack of privacy in bays $(9 \%)$.

Many staff reported in free text feeling grateful and humbled at being able to help those in such difficult situations with what were often very important communications.

Conclusion Data shows that structured virtual visiting has been beneficial for patients \& loved ones during COVID. To address challenges, there is scope for more training provision and addressing connectivity issues. Overall, this is something that would be valuable to continue as good practice, during the Covid-19 pandemic and afterwards.

\section{THE IMPACT OF COVID-19 ON PALLIATIVE CARE AT WIRRAL HOSPICE ST JOHN'S}

Holly Naylor, Martin Gribbin, Emma Longford. Wirral Hospice St John's

\subsection{6/spcare-2021-PCC.33}

Background COVID-19 was declared a pandemic by the World Health Organisation on 12th March 2020. Whilst COVID-19's general impact on health systems is well documented at a national and international level, is as yet unknown how individual hospice-based palliative care services have been affected.

Aims To assess how COVID-19 affected the palliative care service at Wirral Hospice St John's (WHSJ).

Methods The first wave of COVID-19 cases in the UK initially peaked during March, April and May 2020. We collected data from the Hospice's local data system comparing those months in 2020 with 2018 and 2019, as well as longitudinally from September 2018 until present. We looked at inpatient referrals, inpatient length of stay, time from admission to death, outpatient referrals, and outpatient deaths. Secondary outcomes investigated included referral sources to WHSJ outpatient services, and time waiting for a package of care, both measured longitudinally from January 2019 until present.

Results The most significant result found from this study revealed a $64 \%$ drop in average monthly outpatient referrals to the hospice during the 1 st peak of the Coronavirus pandemic, dropping to 17.3 in March to May 2020, compared with 46 in the same monthly period in 2018 and 51.3 in 2019. This trend is even more apparent when viewed over a full two-year timeframe. Further to this, a sharp decline in the number of hospital referrals to outpatient services was seen, dropping from 19 in February to 5 in April 2020.

Discussion/Conclusions The main conclusion that can be drawn from this dataset is that COVID-19 pandemic likely reduced the rate of outpatient referrals to WHSJ. The sizeable reduction is likely multifactorial; due to patients shielding, a reduction in face-to-face GP reviews, reduced hospital admissions for non-COVID-related diseases, wellbeing services unavailability, and a perceived reduction in service capacity. 